\title{
Franck-Condon Factors as Spectral Probes of Polaron Structure
}

\author{
David W. Brown ${ }^{1}$, Aldo H. Romero ${ }^{2}$, and Katja Lindenberg ${ }^{1,3}$ \\ ${ }^{1}$ Institute for Nonlinear Science, \\ University of California, San Diego, La Jolla, CA 92093-0402 \\ 2 Max-Planck Institut für Festkörperforschung, \\ Heisenbergstr. 1, 70569 Stuttgart, Germany \\ ${ }^{3}$ Department of Chemistry and Biochemistry, \\ University of California, San Diego, La Jolla, CA 92093-0340
}

(October 30, 2018)

\begin{abstract}
We apply the Merrifield variational method to the Holstein molecular crystal model in $D$ dimensions to compute nonadiabatic polaron band energies and Franck-Condon factors at general crystal momenta. We analyze these observable properties to extract characteristic features related to polaron selftrapping and potential experimental signatures. These results are combined with others obtained by the Global-Local variational method in $1 \mathrm{D}$ to construct a polaron phase diagram encompassing all degrees of adiabaticity and all electronphonon coupling strengths. The polaron phase diagram so constructed includes disjoint regimes occupied by small polarons, large polarons, and a newly-defined class of compact polarons, all mutually separated by an intermediate regime occupied by transitional structures.
\end{abstract}

PACS numbers: 71.38.+i, 71.15.-m, 71.35.Aa, 72.90.+y

\section{INTRODUCTION}

The theory of polarons has undergone an evolution in recent years that has substantially improved our ability to put solid, quantitative accuracy to matters that have heretofore enjoyed only semi-quantitative estimation or qualitative characterization. This can be said in view of a convergence of results [1] that has been found in a number of independent and high-quality methods that have been brought to bear in particular on the analysis of the Holstein molecular crystal model [2, 3]. Important among these methods are cluster diagonalization [4], density matrix renormalization group [8], quantum Monte Carlo [9 14], and certain variational approaches [1, 15 25]. Though quite distinct in their conception and implementation, these methods have all been found to be in deep and broad quantitative agreement over wide regions of the polaron parameter space.

Our own effort in this area has relied mainly upon the Global-Local variational method, certain results of which will figure in the present work. A significant part of this effort has dealt with the problem of developing a reliable and interpretable polaron phase diagram on which can be clearly delineated the distinct regions of the polaron parameter space where distinct classes of polaron structure may be found. In the course of this development, some familiar notions that have become part of the polaron lore have had to be revised, including the real physical nature of the large polaron 22,23,26 and the meaning of self-trapping in $D$ dimensions [27,28].

Here, we continue to be concerned with the polaron phase diagram, but in a manner and regime that are complementary to what has been already been developed. For practical and formal reasons, the utility of the Global-Local variational method deteriorates significantly when the fundamental electron transfer integrals are small and the electron-phonon coupling is weak; as a practical matter, this limitation excludes a sufficient portion of the non-adiabatic regime to preclude a meaningful assessment of the self-trapping transition there. Necessarily, therefore, what we have been able to say about the polaron phase diagram in the non-adiabatic regime has been based on extrapolations from more adiabatic behaviors.

The non-adiabatic regime is important to many narrow-band systems and particularly to molecular crystals for which the Holstein model was originally formulated [2,3,29 33]. Polaron properties in the non-adiabatic regime generally depend quite smoothly on the basic system parameters, without the relative abruptness that tends to emerge in the adiabatic regime, and the low orders of perturbation theory, either weak-coupling or strong-coupling, tend to do a reasonable job of capturing most behaviors. Paradoxically, perhaps, it is this relative unremarkableness of the non-adiabatic regime that raises some of the questions motivating our study; in particular, how the dramatic character of the self-trapping transition that is so obvious at high adiabaticity dissembles into relative obscurity, and how, as a practical matter, its lingering presence may be recognized in observable polaron properties.

We approach this problem through the use of the Merrifield variational method [18,34]. The Merrifield method can be viewed as an antecedent to the Global-Local method in that it is the first in a sequence of increasingly refined variational methods leading to the GlobalLocal method. Although the Merrifield method suffers some very characteristic limitations that restrict its usefulness as a tool for implementing polaron theory at general points in the polaron parameter space, it is at its best in the non-adiabatic regime where computation by the more general Global-Local method becomes difficult, 
and is thus well-suited to the present task. Moreover, owing to its relative simplicity, it is possible to pursue results in $D$ dimensions, and to obtain some degree of analytical guidance and support for numerical studies.

In order to locate the self-trapping transition, we need to analyze observable physical properties that take on distinguishable asymptotic behaviors on each side of the transition and objectively locate a boundary that discriminates between these behaviors. Here, we focus on two properties that are particularly important to spectral studies, the polaron ground state energy (related in wellknown ways to Stokes shifts) and Franck-Condon factors (related in well-known ways to oscillator strengths). As a function of the electron-phonon coupling strength $g$, the ground state energy generally exhibits a "knee" between distinct weak- and strong-coupling trends that can be located and followed in parameter space to develop a selftrapping line. Franck-Condon factors generally exhibit a distorted Gaussian dependence on the coupling strength, allowing the central peak region (weak coupling) to be objectively distinguished from the outer tail region (strong coupling).

We use the Holstein Hamiltonian [2, 3] on a $D$ dimensional Euclidean lattice

$$
\begin{aligned}
\hat{H}= & -\sum_{\vec{n}} \sum_{i=1}^{D} J_{i} a_{\vec{n}}^{\dagger}\left(a_{\vec{n}+\vec{\epsilon}_{i}}+a_{\vec{n}-\vec{\epsilon}_{i}}\right) \\
& +\hbar \omega \sum_{\vec{n}} b_{\vec{n}}^{\dagger} b_{\vec{n}}-g \hbar \omega \sum_{\vec{n}} a_{\vec{n}}^{\dagger} a_{\vec{n}}\left(b_{\vec{n}}^{\dagger}+b_{\vec{n}}\right),
\end{aligned}
$$

in which $a_{\vec{n}}^{\dagger}$ creates a single electronic excitation in the rigid-lattice Wannier state at site $\vec{n}$, and $b_{\vec{n}}^{\dagger}$ creates a quantum of vibrational energy $\hbar \omega$ in the Einstein oscillator at site $\vec{n}$. The $J_{i}$ are the nearest-neighbor electronic transfer integrals along the primitive crystal axes, and the $\hat{\epsilon}_{i}$ are unit vectors associated with the primitive translations. The above model encompasses all Bravais lattices, with the different lattice structures appearing only in the relative values of the hopping integrals $J_{i}$. For simplicity in the following, we use terms appropriate to orthorhombic lattices in which conventionally $i=x, y$, or $z$; however, all results hold for lattices of lower symmetry with appropriate transcription of these labels to those of the primitive axes.

We use the following Fourier conventions for ladder operators $\left(c^{\dagger}=a^{\dagger}, b^{\dagger}\right)$ and scalars:

$$
\begin{gathered}
c_{\vec{n}}^{\dagger}=N^{-D / 2} \sum_{\vec{p}} e^{-i \vec{p} \cdot \vec{n}} c_{\vec{p}}^{\dagger}, \quad c_{\vec{p}}^{\dagger}=N^{-D / 2} \sum_{\vec{n}} e^{i \vec{p} \cdot \vec{n}} c_{\vec{n}}^{\dagger}, \\
\lambda_{\vec{n}}^{\vec{k}}=N^{-D} \sum_{\vec{q}} e^{i \vec{q} \cdot \vec{n}} \lambda_{\vec{q}}^{\vec{k}}, \quad \lambda_{\vec{q}}^{\vec{k}}=\sum_{\vec{n}} e^{-i \vec{q} \cdot \vec{n}} \lambda_{\vec{n}}^{\vec{k}} .
\end{gathered}
$$

It is convenient in the following to characterize tunneling strength in $D$ dimensions in part through a parameter
$\mathcal{J}=\sum_{i} J_{i}$; when restricting discussion to isotropic tunneling, we use the notation $J=J_{i}$, such that $\mathcal{J}=D J$ in those cases.

For the most part in this paper, we limit our discussion to the non-adiabatic regime, defined by the condition $\mathcal{J} / \hbar \omega<1 / 4$. Polarons at such small $\mathcal{J} / \hbar \omega$ are quite narrow since we know that the largest polaron in any dimension (as characterized by the size of the phonon cloud) has a width of $\sqrt{2 J_{i} / \hbar \omega}$ along the $i$ axis [26]; since no $J_{i} / \hbar \omega$ is greater than $1 / 4$ in the non-adiabatic regime, no polaron in this regime has a width greater than a lattice constant, even at vanishing coupling. Thus, the variational space in which the problem is solved numerically need not be large in order to contain the complete polaron. This ability to contain the present problem in a small real-space volume facilitates computation considerably.

The Merrifield trial state may be written

$$
\begin{aligned}
&|\Psi(\vec{\kappa})\rangle= N^{-D / 2} \sum_{\vec{n}} e^{i \vec{\kappa} \cdot \vec{n}} a_{\vec{n}}^{\dagger} \\
& \times \exp \left[-N^{-D / 2} \sum_{\vec{q}}\left(\lambda_{\vec{q}}^{\vec{\kappa}} e^{-i \vec{q} \cdot \vec{n}} b_{\vec{q}}^{\dagger}-h . c .\right)\right]|0\rangle, \\
&\left\langle\Psi(\vec{\kappa}) \mid \Psi\left(\vec{\kappa}^{\prime}\right)\right\rangle=\delta_{\vec{\kappa}^{\prime}},
\end{aligned}
$$

in which the $\left\{\lambda_{\vec{q}}^{\vec{\kappa}}\right\}$ are the variational parameters specifying the coherent state amplitude in the phonon mode $\vec{q}$. Though these trial states are delocalized and satisfy the appropriate Bloch symmetry condition, and thus any property measured in the "lab" frame is uniform over the lattice, the internal structure of these delocalized states is determined by exciton-phonon correlations that are essentially local in character. Here, that local character is such that the electronic component located at site $\vec{n}$ is associated with a "phonon cloud" centered on that site, determined by the set of lattice amplitudes $\left\{\lambda_{\vec{q}}^{\vec{\kappa}}\right\}$.

We evaluate the variational energy band as the expectation value of the Holstein Hamiltonian

$$
\begin{aligned}
E^{\vec{\kappa}}= & \langle\Psi(\vec{\kappa})|\hat{H}| \Psi(\vec{\kappa})\rangle=-\sum_{i=1}^{D} J_{i}\left(e^{i \kappa_{i}} S_{+i}^{\vec{\kappa} *}+e^{-i \kappa_{i}} S_{-i}^{\vec{\kappa}}\right) \\
& +N^{-D} \hbar \omega \sum_{\vec{q}}\left|\lambda_{\vec{q}}^{\vec{\kappa}}\right|^{2}-N^{-D} g \hbar \omega \sum_{\vec{q}}\left(\lambda_{-\vec{q}}^{\vec{\kappa} *}+\lambda_{\vec{q}}^{\vec{\kappa}}\right),
\end{aligned}
$$

wherein $S_{ \pm i}^{\vec{\kappa}}$ is the Debye-Waller factor

$$
S_{ \pm i}^{\vec{\kappa}}=\exp \left[N^{-D} \sum_{\vec{q}}\left|\lambda_{\vec{q}}^{\vec{k}}\right|^{2}\left(e^{ \pm i q_{i}}-1\right)\right]
$$

Minimization of $E^{\vec{\kappa}}$ with respect to $\lambda_{\vec{q}}^{\vec{\kappa} *}$ leads to the self-consistency equations for $\lambda_{\vec{q}}^{\vec{k}}$ :

$$
\begin{gathered}
\lambda_{\vec{q}}^{\vec{r}}=\frac{g \hbar \omega}{\hbar \omega-\sum_{i=1}^{D}\left[4 J_{i} S_{i}^{\vec{\kappa}} \sin \left(\kappa_{i}-\Phi_{i}^{\vec{\kappa}}-\frac{q_{i}}{2}\right) \sin \frac{q_{i}}{2}\right]}, \\
S_{i}^{\vec{\kappa}}=\exp \left[N^{-D} \sum_{\vec{q}}\left|\lambda_{\vec{q}}^{\vec{\kappa}}\right|^{2}\left(\cos q_{i}-1\right)\right]
\end{gathered}
$$




$$
\begin{gathered}
\Phi_{i}^{\vec{\kappa}}=N^{-D} \sum_{\vec{q}}\left|\lambda_{\vec{q}}^{\vec{\kappa}}\right|^{2} \sin q_{i}, \\
S_{i}^{\vec{\kappa}}=S_{i}^{-\vec{\kappa}}, \quad \Phi_{i}^{\vec{\kappa}}=-\Phi_{i}^{-\vec{\kappa}}, \quad \lambda_{\vec{q}}^{\vec{k}}=\lambda_{-\vec{q}}^{-\vec{k}},
\end{gathered}
$$

where $S_{i}^{\vec{\kappa}}$ and $\pm \Phi_{i}^{\vec{\kappa}}$ are the magnitudes and phases of the complex Debye-Waller factors $S_{ \pm i}^{\vec{r}}$. This shows the optimal $\lambda_{\vec{q}}^{\vec{\kappa}}$ to be real, and establishes the "sum rule"

$$
\sum_{\vec{n}} \lambda_{\vec{n}}^{\vec{\kappa}}=\lambda_{\vec{q}=0}^{\vec{k}}=g
$$

valid at any $\vec{\kappa}$ and in any number of dimensions.

When any particular $J_{i} / \hbar \omega \rightarrow 0, \lambda_{\vec{q}}^{\vec{R}}$ loses any dependence on $q_{i}$ and $\kappa_{i}$, becoming "flat" in those variables. The Debye-Waller factor $\left(S_{i}^{\vec{\kappa}}\right)$ and phase $\left(\Phi_{i}^{\vec{\kappa}}\right)$ associated with that direction drop out of the problem and the real space phonon amplitudes $\lambda_{\vec{n}}^{\vec{k}}$ become completely localized along the $i$ axis. Although a disparity among the relative magnitudes of several $J_{i}$ can result in a polaron that is in respects "small" in certain directions and "large" in others, there is not a distinct self-trapping transition associated with each $J_{i}$ [28]. This can be seen in the present context in the fact that the Debye-Waller factor $S_{z}^{\vec{\kappa}}$ associated with a vanishing $J_{z}$ does not approach $e^{-g^{2}}$, which would be expected of $1 \mathrm{D}$ small polarons along the $z$ axis, but a quantity that is characteristic of the $2 \mathrm{D}$ polaron structure in the two surviving dimensions, whether this be large-polaron-like or small-polaron-like.

The sum rule continues to be satisfied as dimensions are turned off or on (e.g., $J_{z} \rightarrow 0$ in three dimensions), since

$$
\begin{aligned}
\lim _{J_{z} \rightarrow 0} & \sum_{n_{x}, n_{y}, n_{z}} \lambda_{\left(n_{x}, n_{y}, n_{z}\right)}^{\left(\kappa_{x}, \kappa_{y}, \kappa_{z}\right)}= \\
& \sum_{n_{x}, n_{y}, n_{z}} \lambda_{\left(n_{x}, n_{y}\right)}^{\left(\kappa_{x}, \kappa_{y}\right)} \delta_{n_{z}, 0}=\sum_{n_{x}, n_{y}} \lambda_{\left(n_{x}, n_{y}\right)}^{\left(\kappa_{x}, \kappa_{y}\right)}=g .
\end{aligned}
$$

Thus, there is no need for dimension-specific formulation if dimensions are controlled through the tuning of $\left\{J_{i} / \hbar \omega\right\}$.

Owing to the symmetries (11) and the periodicity of the reciprocal lattice, $\Phi_{i}^{\vec{\kappa}}$ vanishes at the Brillouin zone center and everywhere on the Brillouin zone boundary. Consequently, we have certain special values that play a significant role in the following. Denoting the reciprocal lattice origin by $\overrightarrow{0}$ and any of the most extreme points of the Brillouin zone by $\vec{\pi}\left(\kappa_{i}= \pm \pi\right.$ along each axis), we find

$$
\begin{aligned}
& \lambda_{\vec{q}}^{\overrightarrow{0}}=\frac{g \hbar \omega}{\hbar \omega+\sum_{i=1}^{D}\left[4 J_{i} S_{i}^{\overrightarrow{0}} \sin ^{2} \frac{q_{i}}{2}\right]}, \\
& \lambda_{\vec{q}}^{\vec{\pi}}=\frac{g \hbar \omega}{\hbar \omega-\sum_{i=1}^{D}\left[4 J_{i} S_{i}^{\vec{\pi}} \sin ^{2} \frac{q_{i}}{2}\right]} .
\end{aligned}
$$

The zone-center phonon amplitudes (14) are wellbehaved under all circumstances because the denominators, similar to those of weak-coupling perturbation theory, are sums of bounded positive terms. The zone-edge relation (15), on the other hand, suggests the possibility of encountering large or divergent phonon amplitudes for phonon wave vectors near the Brillouin zone boundary if tunneling is sufficiently strong $(\mathcal{J} / \hbar \omega \geq 1 / 4)$ and the Debye-Waller factors $\left\{S_{i}^{\vec{\pi}}\right\}$ are sufficiently near unity (as generally occurs when electron-phonon coupling is sufficiently weak; see the 1D examples below). This potential divergence is both a real physical phenomenon and a generator of artifacts in the Merrifield method.

The reality of the phenomenon is due to the resonance or near-resonance that can occur between the states of the one-phonon continuum and zone-edge states of both the free-electron and the self-consistent polaron when the energy gap between latter and the one-phonon continuum is small. This circumstance occurs in any number of dimensions when $\mathcal{J} / \hbar \omega \gtrsim 1 / 4$ and $g$ is small. Under these circumstances, only a very small amount of electron-phonon coupling is needed to produce intense interactions that flatten the outer polaron energy band (level repulsion) and create heavy phonon clouds strongly modulated by the character of the zone-edge phonons.

The Merrifield method accommodates the nearness of the one-phonon continuum by producing strong distortions of the variational amplitudes of a qualitatively appropriate nature; the phonon amplitudes become highly focussed around a single phonon wave vector, in clear approximation to the single-phonon quantum that constitutes the exact $g \rightarrow 0$ state. However, because the Merrifield state is not well-equipped to emulate the highly quantum mechanical character of such states, the energy balance central to the variational principle is distorted and the variational energy bound is raised. Consequently, rather than experiencing the expected strong repulsion from the one-phonon continuum, the outer-zone portions of Merrifield energy bands flatten relatively weakly and cannot be taken as appropriately indicative of the polaron structure when $\mathcal{J} / \hbar \omega \gtrsim 1 / 4$.

Although one cannot rely upon the numerical values of Merrifield band energies influenced by resonances with the one-phonon continuum, it is nonetheless true that the general character of the variational lattice state responds to such resonance effects in a reasonably appropriate way, provided that $\mathcal{J} / \hbar \omega \leq 1 / 4$. This suggests that changes in the variationally-determined Franck-Condon factors, as very direct figures of merit for this general character, may reasonably indicate where the essential changes in polaron structure occur. It is thus that in the following we rely upon Franck-Condon factors as our primary diagnostic of outer-zone polaron structure. Although this proves to be a very practical election, it is a choice that is in respects forced upon us by the limitations of the Merrifield method. As a choice that in the larger picture should be sufficient, but not necessary, other theoretical methods not so limited should find similar behavior in 
the band energies and other polaron properties near the band edge.

\section{THE 1D CASE}

In principle, the set of equations (8) - (10) can be closed in the $S_{i}^{\vec{\kappa}}$ and $\Phi_{i}^{\vec{\kappa}}$ alone, greatly reducing the size of the self-consistency problem to be solved. This is of practical advantage only in one dimension, however, since the reduction to quadratures involved in higher dimensions does not significantly facilitate computation.

Replacing the summations in Eqs. (9) and (10) with 1D integrations, one arrives at the self-consistency equations first obtained by Merrifield:

$$
\begin{gathered}
S^{\kappa}=\exp \left(-g^{2} \Delta^{\kappa}\right), \\
\Phi^{\kappa}=-g^{2} \Delta^{\kappa}\left(2 J S^{\kappa} / \hbar \omega\right) \sin \left(\Phi^{\kappa}-\kappa\right),
\end{gathered}
$$

and

$$
\Delta^{\kappa}=\left[\frac{\hbar \omega}{\sqrt{\left[\hbar \omega+2 J S^{\kappa} \cos \left(\Phi^{\kappa}-\kappa\right)\right]^{2}-\left(2 J S^{\kappa}\right)^{2}}}\right]^{3} .
$$

Using (16) - (18) in (8) yields the full set of variational phonon amplitudes such as shown in Figure 1 .

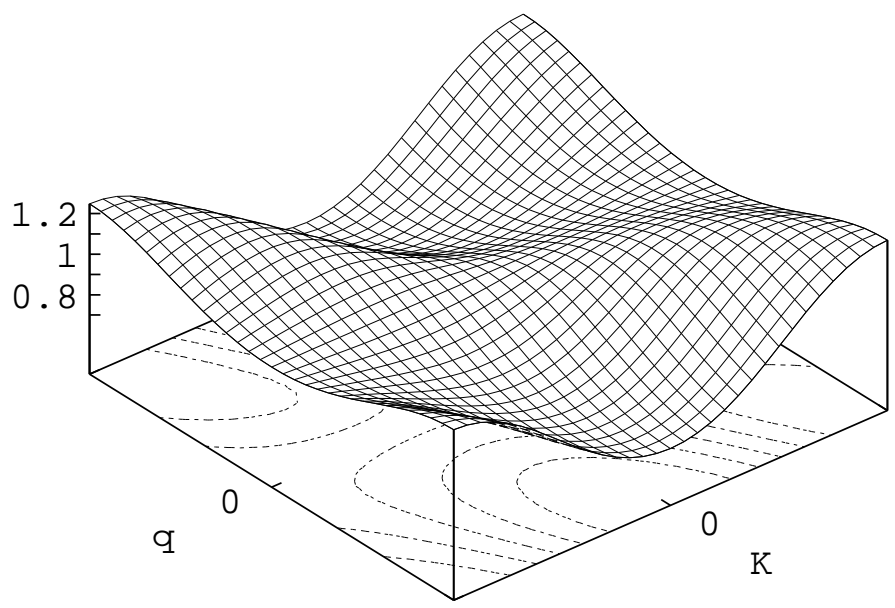

FIG. 1. Sample surface showing the (real) variational amplitudes $\lambda_{q}^{\kappa}$ in the $1 \mathrm{D}$ case for $J / \hbar \omega=0.2$ and $g=1$.

One may further obtain the energy-momentum relation:

$$
\begin{aligned}
E^{\kappa}= & g^{2} \hbar \omega\left(\Delta^{\kappa}-2\left[\Delta^{\kappa}\right]^{\frac{1}{3}}\right) \\
& -2 J S^{\kappa}\left(1-g^{2} \Delta^{\kappa}\right) \cos \left(\kappa-\Phi^{\kappa}\right) .
\end{aligned}
$$

The price paid for the compactness of this expression is the self-consistency condition that makes (19) awkward to analyze; however, it can be shown that (19) agrees with weak-coupling perturbation theory through second order in $g$, and strong-coupling perturbation theory through first order in $J / \hbar \omega$. The latter is actually a shortcoming of the Merrifield method since important contributions from second order quickly dominate the first order of strong-coupling perturbation theory; however, the first order is sufficient to properly determine that in the $J / \hbar \omega \rightarrow 0$ limit the "knee" in the dependence of $E^{\kappa}$ on $g$ at any $\kappa$ is given by

$$
\frac{\partial^{3} E^{\kappa}}{\partial g^{3}}=0 \Rightarrow g=\sqrt{\frac{3}{2}} \text { at } \frac{J}{\hbar \omega}=0
$$

The same differential criterion can be applied at finite $J / \hbar \omega$, which we will use to develop the phase diagram in Section IV below.

\section{THE FRANCK-CONDON FACTOR}

A quantity intimately related to the Debye-Waller factors appearing in the self-consistency equations is the Franck-Condon factor

$$
F(\vec{\kappa})=\left|\left\langle\Psi(\vec{\kappa})\left|a_{\vec{\kappa}}^{\dagger}\right| 0\right\rangle\right|^{2} .
$$

This is one of many Franck-Condon factors associated with various transitions between correlated electronphonon states. This particular factor characterizes the oscillator strength of the zero-phonon line associated with a transition between a free electron state of crystal momentum $\vec{\kappa}$ and the polaron it forms at the same $\vec{\kappa}$; these are direct transitions, resolved by crystal momentum. Though one is often concerned primarily with transitions near the Brillouin zone center, we will use the full $\vec{\kappa}$ dependence of $F(\vec{\kappa})$ across the Brillouin zone, and particular at the zone center and the zone boundary.

Our principal interest in zero-phonon lines in the present context is in the possibility that they may offer an observable means for mapping the essential polaron features on the polaron phase diagram. Owing to the strong similarity between the Franck-Condon factor and the Debye-Waller factors intimately connected with the polaron effective mass, it is reasonable to expect that an analysis of the dependence of the Franck-Condon factor on model parameters should be able to yield the location of the self-trapping line. Also owing to the fact that the Merrifield method is at its best in the non-adiabatic regime and weak coupling, it is reasonable to hope that such an analysis would complement others made by other methods generally more accurate (e.g., the Global-Local method), but which deteriorate in quality at small $\mathcal{J} / \hbar \omega$ and $g$.

The quenching of the zero-phonon line is an experimental signature that has long been associated with the selftrapping transition. As a function of model parameters, this quenching occurs continuously as electron-phonon coupling is tuned from the weak-coupling to the strongcoupling regimes. There is thus some inherent ambiguity 
in the assignment of the point we associate with the selftrapping transition; our criterion here, as elsewhere, is to identify the self-trapping transition as the point of most rapid change in a property that takes on characteristically different behaviors in the weak and strong-coupling regimes. In the case of the Franck-Condon factor, this criterion takes the form of an inflection point in the dependence of $F(\vec{\kappa})$ on $g$ at fixed $\left\{J_{i}\right\}$.

In terms specific to our variational development,

$$
F(\vec{\kappa})=\exp \left(-N^{-D} \sum_{\vec{q}}\left|\lambda_{\vec{q}}^{\vec{\kappa}}\right|^{2}\right),
$$

which is just the exponential of the average number of phonons per mode in the phonon cloud.

In the $1 \mathrm{D}$ case, we find

$$
F(\kappa)=\exp \left\{-g^{2} \Delta^{\kappa}\left[1-\left(2 J S^{\kappa} / \hbar \omega\right) \cos \left(\kappa-\Phi^{\kappa}\right)\right]\right\},
$$

as shown in Figure 2 in selected cases.

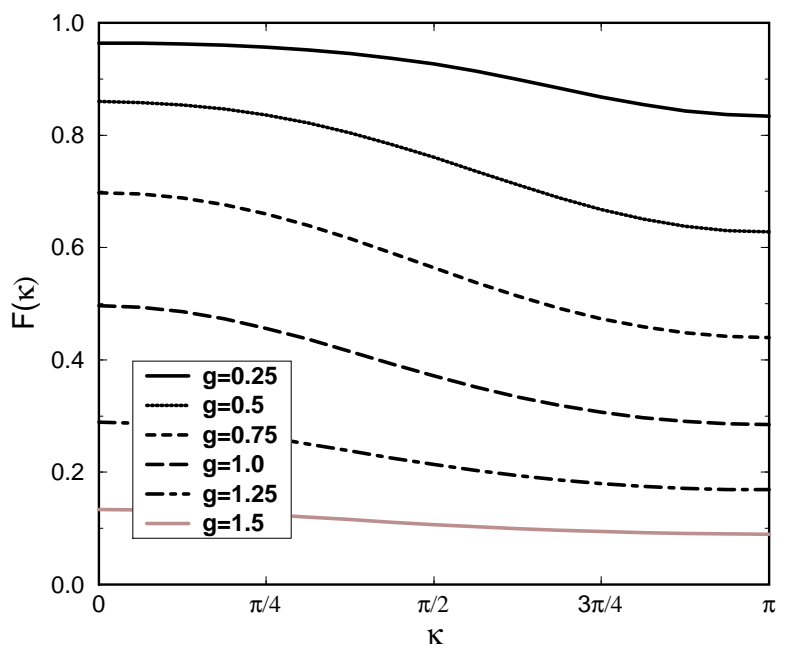

FIG. 2. Franck-Condon factors across the Brillouin zone in $1 \mathrm{D}$. $J / \hbar \omega=0.2, g=0.25-1.5$.

It is simple to show that in the absence of tunneling $F(\vec{\kappa})=e^{-g^{2}}$ for all $\vec{\kappa}$ in any number of dimensions. Thus, in the $\mathcal{J} / \hbar \omega \rightarrow 0$ limit the self-trapping features associated with the Franck-Condon factor are found at $g=1 / \sqrt{2}$, for any $\vec{\kappa}$. With increasing tunneling, this degeneracy is broken and these features fan out; the manner in which this occurs can be seen most clearly in the 1D case, where

$$
\begin{aligned}
& F(\kappa=0)=\exp \left\{-g^{2} \frac{1-2 J S^{0} / \hbar \omega}{\left(1+4 J S^{0} / \hbar \omega\right)^{3 / 2}}\right\}, \\
& F(\kappa=\pi)=\exp \left\{-g^{2} \frac{1+2 J S^{\pi} / \hbar \omega}{\left(1-4 J S^{\pi} / \hbar \omega\right)^{3 / 2}}\right\} .
\end{aligned}
$$

(See Figure 3.) It is clear from these that the FranckCondon factor is independent of $\kappa$ at $J / \hbar \omega=0$. It is also clear that increasing $J / \hbar \omega$ from 0 causes $F(0)$ to broaden out to stronger coupling and causes $F(\pi)$ to narrow toward weaker coupling.

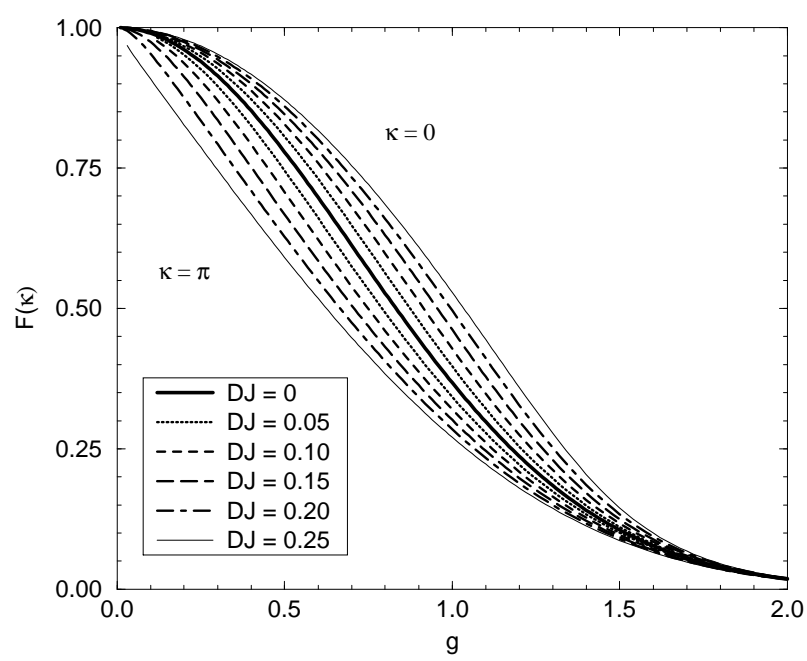

FIG. 3. Franck-Condon factors in $D$ dimensions at the zone center (upper curves) and at the most extreme point of the Brillouin zone $\left(\kappa_{i}=\pi\right)$ for $D J / \hbar \omega=0-0.25$.

Perhaps the most interesting behavior revealed in (23) - (25) is that of $F(\pi)$ at $J / \hbar \omega=1 / 4$. Using the fact that at this particular $J / \hbar \omega$ value

$$
S^{\pi}=\exp \left[-g^{2}\left(1-S^{\pi}\right)^{-3 / 2}\right],
$$

one can show that the leading dependence of $S^{\pi}$ on $g$ is

$$
S^{\pi} \sim 1-g^{4 / 5}
$$

This in turn implies that in the same approximation,

$$
F(\pi) \sim \exp \left[-\frac{3}{2} g^{4 / 5}\right]
$$

(See Figure A.) This singular behavior in the FranckCondon factor at $J / \hbar \omega=1 / 4$ suggests that the selftrapping feature identified by an inflection point in $g$ moves to $g=0$ at $J / \hbar \omega=1 / 4$. Indeed, on can show that for $J / \hbar \omega=1 / 4-\epsilon$, the Franck-Condon factor retains an initial finite negative curvature in $g$, suggesting that there exists a proper inflection point at finite $g$. 


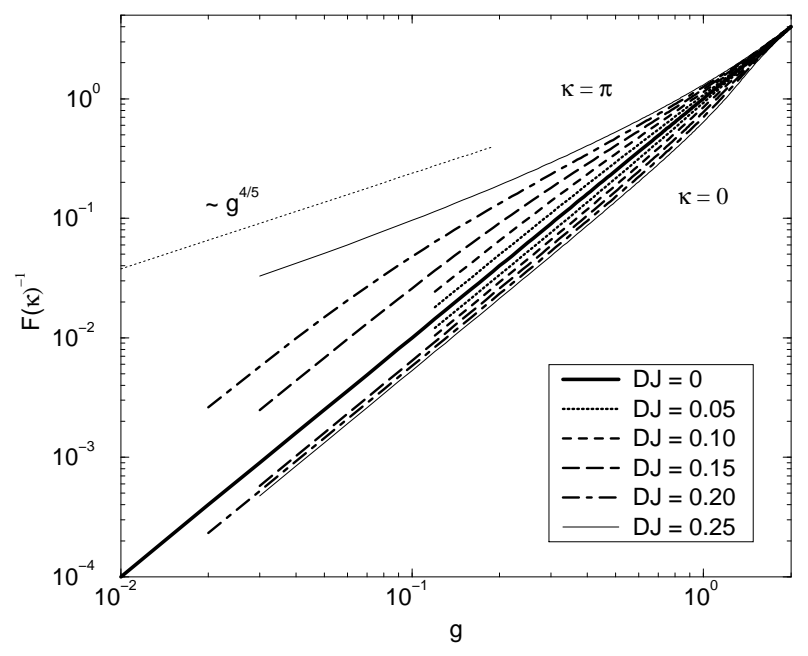

FIG. 4. $\log (-\ln F(\vec{\kappa}))$ vs. $\log g$, allowing the power of $g$ in the exponent of the Franck-Condon factor to be ascertained. Curves are coded to correspond to Figure 3; zone center (lower curves) and zone edge (upper curves) in $D$ dimensions for $D J / \hbar \omega=0,0.05,0.1,0.15,0.2$, and 0.25 .

Thus, all these considerations suggest that the selftrapping feature in $F(0)$ should should shift more-or-less steadily from $g=1 / \sqrt{2}$ at $J / \hbar \omega=0$ to stronger coupling with increasing $J / \hbar \omega$, while the self-trapping feature in $F(\pi)$ appears to shift from the same $g=1 / \sqrt{2}$ to weaker coupling with increasing $J / \hbar \omega$, arriving at $g=0$ at $J / \hbar \omega=1 / 4$.

The particular results above have been obtained for the 1D case, which enjoys sufficient tractibility to admit some reasonably straightforward formal analysis. In higher dimensions, numerical solution and analysis is generally more practical, though certain exceptions warrant special attention. Throughout the foregoing we have highlighted the particular crystal momentum values associated with the Brillouin zone center $(\vec{\kappa}=\overrightarrow{0})$ and the most remote corners of the Brillouin zone where all the crystal momentum components take their maximum values $(\vec{\kappa}=\vec{\pi})$. We demonstrate in Appendix A that for these particular $\vec{\kappa}$ values in the isotropic cases in $D$ dimensions, the dependence of the Franck-Condon factors on the dimensionality and tunneling strength is reduced to the single scaled variable $D J$. This implies that the particular results shown in Figure 3 hold not only in 1D, but in 2D and $3 \mathrm{D}$ as well when parameters are scaled appropriately.

Results can be obtained numerically for any degree of anisotropy and general $\vec{\kappa}$; however, the qualitative nature of the dependence on anisotropy can be inferred from Figure 3 without detailed analysis. Consider, for example, $\vec{\kappa}=\overrightarrow{0}$ and $J / \hbar \omega=0.05$ : The results for quasi-2D scenarios with $J_{x} / \hbar \omega=J_{y} / \hbar \omega=0.05$ and $0<J_{z} / \hbar \omega<0.05$ are contained between the
$D J / \hbar \omega=0.15$ and $D J / \hbar \omega=0.1$ cases shown in Figure 3. Similarly, the results for quasi-1D scenarios with $J_{x} / \hbar \omega=0.05,0<J_{y} / \hbar \omega<0.1$ and $J_{z} / \hbar \omega=0$ are contained between the $D J / \hbar \omega=0.05$ and $D J / \hbar \omega=0.1$ cases shown in Figure 3 .

The tunneling parameters and the effective dimensionality determined by them are not generally subject to any practical degree of experimental control; however, even greater changes in the Franck-Condon factor can be induced by changing the magnitude and/or the orientation of the crystal momentum $\vec{\kappa}$ probed. To the extent that it is possible to achieve some selectivity in the $\vec{\kappa}$ 's sampled in a particular experiment, it should be possible to induce controlled variations in the oscillator strengths associated with these Franck-Condon factors by, for example, varying the orientation of the sample. Such predictable "wobbles" in the intensities of appropriately-selective spectral probes constitute signatures of polaron structure that exist only if both electron-phonon coupling and electron tunneling are sufficiently great, without reducing the quasiparticle to the status of a "mere" small polaron.

\section{PHASE DIAGRAM}

The overall character of the foregoing results can be summarized on a diagram of the polaron parameter space in which the loci of the knees in the polaron band energies and the inflection points of Franck-Condon factors play the role of rough phase boundaries separating distinct classes of polaron structure (see Figure 5). These lines are accurately described by the simple relations

$$
\begin{array}{rlrl}
g_{E^{0}} & \sim \sqrt{\frac{3}{2}}\left(1+\frac{2}{3} \frac{D J}{\hbar \omega}\right), & & D J / \hbar \omega \lesssim 1 / 4, \\
g_{E^{\pi}} & \sim \sqrt{\frac{3}{2}}\left(1-\frac{2}{3} \frac{D J}{\hbar \omega}\right), & D J / \hbar \omega \ll 1 / 4, \\
g_{F^{0}} \sim \frac{1}{\sqrt{2}}\left(1+4 \frac{D J}{\hbar \omega}\right)^{2 / 3}, & D J / \hbar \omega \lesssim 1 / 4, \\
g_{F^{\pi}} \sim \frac{1}{\sqrt{2}}\left(1-4 \frac{D J}{\hbar \omega}\right)^{1 / 2}, & D J / \hbar \omega<1 / 4 .
\end{array}
$$

obtained in empirical fashion by noting the exact $\mathcal{J}=0$ termini as discussed in prior sections and augmenting these with the simplest expressions in whole numbers that express the apparent trends in a quantitatively consistent way. The restrictions on (29) and (31) are weak because such zone-center properties are well-behaved under the Merrifield method to $\mathcal{J} / \hbar \omega$ substantially greater than $1 / 4$; however, the quantitative accuracy of the Merrifield method even at the zone center deteriorates significantly with increasing $\mathcal{J} / \hbar \omega$, warranting prudence beyond the strictly non-adiabatic regime. On the other hand, the restrictions on (30) and (32) are strong because zone-edge properties are strongly affected by the one-phonon continuum. 
These boundaries only roughly distinguish distinct polaron regimes because the structural changes occurring in the non-adiabatic regime are quite smooth and broad, with changes in different aspects of polaron structure occurring with less synchronization than is seen in the adiabatic regime. This is seen clearly in the fact that the critical features of the band energies and the FranckCondon factors are significantly separated in $g$ in the $\mathcal{J} / \hbar \omega \rightarrow 0$ limit, and while trending similarly with increasing $\mathcal{J} / \hbar \omega$, remain well separated over the entire non-adiabatic regime.

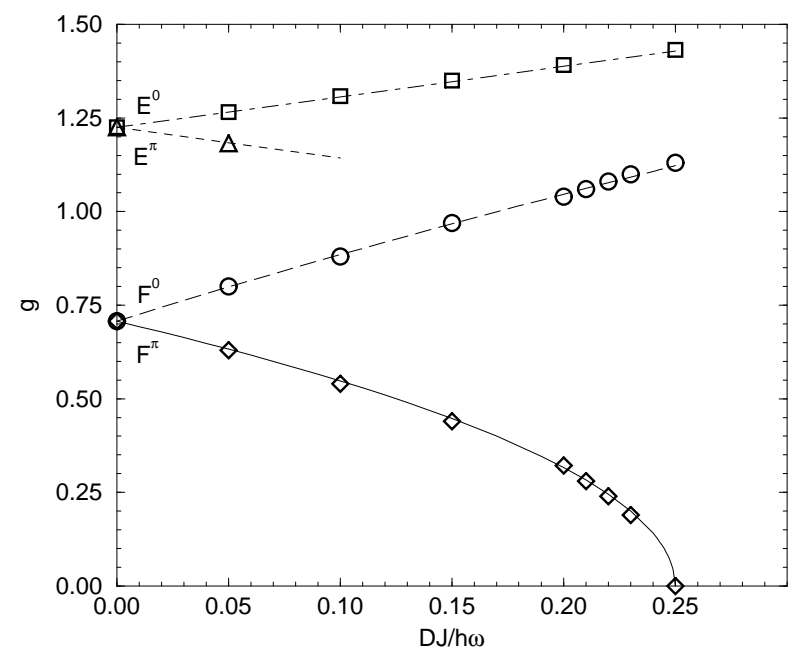

FIG. 5. Polaron phase diagram in the non-adiabatic regime according to the Merrifield method in $D$ dimensions. Diamonds: Location of inflection points in $F(\pi)$. Circles: Location of inflection points in $F(0)$. Squares: Location of the knee in $E^{0}$. Triangles: Location of the knee in $E^{\pi}$. Solid line, Eq. 32. Long-dashed line, Eq. 31. Short-dashed line, Eq. 30. Chain-dotted line, Eq. 29.

This disperse character of the collection of selftrapping-related loci is not an artifact of the Merrifield method, nor of the particular parameter regime nor of the particular physical quantities used to locate transition effects. A similar and complementary dispersion has been found at somewhat larger $J / \hbar \omega$ in 1D using the Global-Local method, based on the analysis of physical quantities such as the ground state energy, kinetic energy, phonon energy, electron-phonon interaction energy [20], effective mass [21], and electron-phonon correlation functions [22]. In such analyses, the self-trapping loci attributable to different zone-center physical quantities have been found to cluster increasingly tightly with increasing $J / \hbar \omega$, permitting an empirical self-trapping curve

$$
g_{S T}=1+\sqrt{\frac{J}{\hbar \omega}}
$$

to be identified that appears to accurately describe the central trend of such clusters of data over essentially the entire adiabatic regime.

Similarly, a zone-edge curve

$$
g_{N}=1+\sqrt{\frac{J}{\hbar \omega}}-\left[8\left(\frac{J}{\hbar \omega}-\frac{1}{4}\right)+\left(\frac{2}{3}\right)^{8}\right]^{-1 / 8}
$$

in $1 \mathrm{D}$ has been identified that appears to accurately describe the characteristic changes in the outer energy band that signal the onset of significant narrowing of the polaron energy band, commencing the process that develops into the self-trapping transition with increasing coupling strength.

It is telling to combine the empirical curves (33) and (34) abstracted from our prior 1D Global-Local analyses with the complementary curves (29), (31), and (32) that follow from our present analysis by the Merrifield method. This comparison is presented in Figure 6.

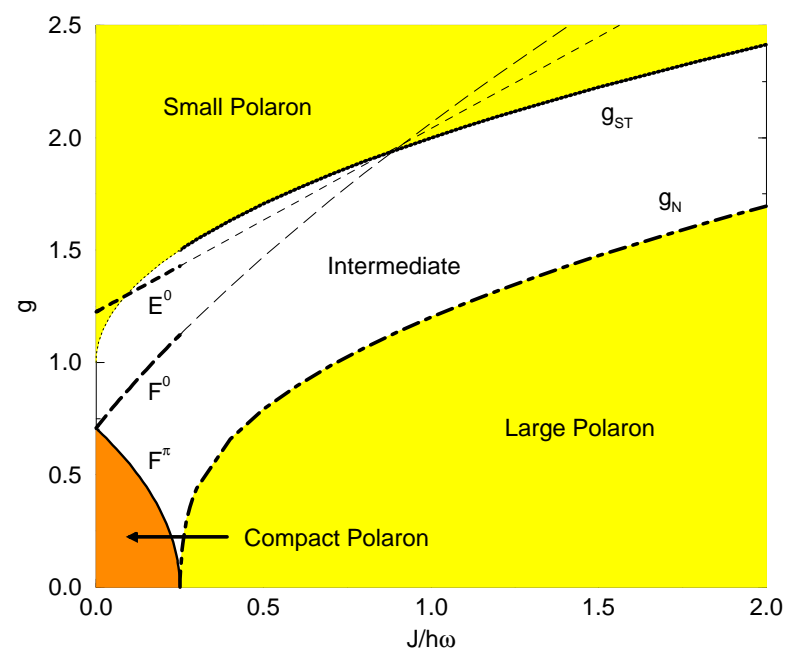

FIG. 6. Polaron phase diagram combining the empirical curves (29), (31), and (32) based on the present analysis employing the Merrifield method for $D J / \hbar \omega<1 / 4$ with the complementary empirical curves (33) and (34) based on independent analyses by the Global-Local variational method for $J / \hbar \omega>1 / 4$ in $1 \mathrm{D}$.

It is clear that zone-center properties follow a common pattern of behavior, relatively indifferent to the crossover from the non-adiabatic into the adiabatic regime but for the possible role the latter may play in setting the scale over which the disperse self-trapping loci of the nonadiabatic regime begin to cluster more tightly toward the more sharply-defined trend in the adiabatic regime. This dispersity does not vanish suddenly at the crossover, but is apparent as well in the dispersity of similar loci found under the Global-Local method at small $J / \hbar \omega$ still greater than $1 / 4$. 
Further, it is interesting and surely no accident that the Merrifield zone-center lines (29) and (31) intersect, that this intersection falls very nearly upon the GlobalLocal zone-center line (33), and that this cluster of intersections coincides well with the first appearance of discontinuities in the solutions of the Merrifield method. It was, in fact, the set of such "critical points" $\left(J_{c} / \hbar \omega, g_{c}\right)$ ascertained from our sequentially-refined variational calculations (Merrifield method [18], Toyozawa method [19], Global-Local method [16]) that first led us to identify the simple empirical curve (33) as a convenient method-independent approximant to the real physicallymeaningful self-trapping line. The "critical" appearance of solutions near such points $\left(J_{c} / \hbar \omega, g_{c}\right)$ is, of course, a methodological artifact, and we should view the intersections of the several zone-center lines near $J / \hbar \omega \approx 0.9$ as an artifact as well; the physical self-trapping line surely bends smoothly through this region, seamlessly joining the central trend of the non-adiabatic loci with the more sharply-defined trend that develops at higher adiabaticity.

These zone-center results of the Merrifield method suggest an answer to one of the more empirical questions left open by our prior Global-Local analyses. Though quite accurate over a large range of $J / \hbar \omega$, it has seemed unlikely that the dependence of the empirical curve $g_{S T}$ on $\sqrt{J / \hbar \omega}$ should continue unregularized all the way down to $J / \hbar \omega=0$. If we are to continue to regard the physically meaningful $g_{S T}$ as representing a central trend in the inherently disperse set of self-trapping loci even as $J / \hbar \omega$ vanishes, the present results suggest that the leading dependence of $g_{S T}$ on $J / \hbar \omega$ should not persist as a square root, but yield to a more pedestrian linear dependence

$$
g_{S T} \sim 1+a \frac{J}{\hbar \omega}, \quad \frac{J}{\hbar \omega}<\frac{1}{4}
$$

where $a$ is a constant of order unity.

It is likewise clear that the zone-edge loci follow a common pattern of behavior, albeit one that is exquisitely sensitive to the crossover from the non-adiabatic into the adiabatic regime. Although the loci illustrated above and below this crossover are drawn from different physical properties (Franck-Condon factors from the Global-Local method are not available and the Merrifield band energies near the zone edge are not meaningful in and beyond the crossover regime), they are closely related and reflect the same, if complementary, underlying physical behavior.

We are led to view the results of the Merrifield method in the non-adiabatic regime and those of the Global-Local method in the adiabatic regime as mutually confirming, and describing one consistent set of self-trapping phenomena at all $J / \hbar \omega$.

In the adiabatic regime, it is quite straightforward to view the two lines $g_{S T}$ and $g_{N}$ as dividing the polaron parameter space into a small polaron regime at strong coupling, a large-polaron regime at weak coupling, and an intermediate regime occupied by transitional structures.
In the non-adiabatic regime, it likewise clear that there is an unambiguous small polaron regime at strong coupling; moreover, it is noteworthy that the non-adiabatic small polaron states are continuously deformable into the adiabatic small polaron states without encountering transition behavior in any basic polaron property, so that there is no formal distinction to be made between nonadiabatic and adiabatic small polarons. A complementary observation can be made about large polarons; although large polarons as we have thus far characterized them reside in the adiabatic regime, there is no formal distinction to be drawn between large polarons at greater or lesser electron-phonon coupling strengths since these are continuously deformable into each other without encountering any transition behavior. The intermediate regime can be defined in similar terms as that in which transition behavior is found in some basic polaron property at every point; for example, although the lines $g_{S T}$ (zone center) and $g_{N}$ (zone edge) are only discrete curves, the domain between them is dense with similar curves associated with the occurrence of transition behavior at general $\kappa$ values.

These observations lead us to consider the more darklyshaded region of Figure 6 at the weak-coupling end of the non-adiabatic regime. The transition line 32 associated with the zone-edge Franck-Condon factor and the more limited, qualitative information available in the Merrifield band-edge energies (30) suggests that this regime is disconnected from both the small polaron regime and the large polaron regime in the sense of continuous deformability as used above. Provided that this non-adiabatic weak-coupling regime is not dense with transition loci, which seems quite unlikely, it would appear that this regime is occupied by a polaron structure that is neither "small" nor "large" nor of a transitional nature that would identify it with the intermediate regime.

Indeed, some very basic polaron properties behave in qualitatively distinct ways in this insular regime, perhaps foremost being the polaron radius as given by the polaron Wannier function. Intuitively, one expects the polaron radius by any definition to decrease monotonically with increasing electron-phonon coupling, and this is generally the case in the regimes we have here characterized as the small polaron regime and the large polaron regime. Even in the non-adiabatic weak-coupling regime now under discussion, the radius of the phonon cloud associated with zone-center polarons has an initial width of order $\sqrt{2 J_{i} / \hbar \omega}$ along the $i$ axis 26 , and decreases with increasing coupling strength.

The polaron Wannier function, however, is a construct of the entire polaron energy band, being a superposition of polaron Bloch states of every $\vec{\kappa}$; as such, it is a localized state that can be viewed as energy band theory's own answer to the inverse problem of determining the identity of the localized quasiparticle whose dynamical properties are manifested in the polaron energy band. The realspace width of this state can be gauged in various ways, among them being a variance measure of the electron 
density within the polaron Wannier state.

In present terms, we may construct polaron Wannier states from the trial Bloch states in the following fashion

$$
|\Phi(\vec{n})\rangle=\frac{1}{\sqrt{N^{D}}} \sum_{\vec{\kappa}} e^{-i \vec{\kappa} \cdot \vec{n}}|\Psi(\vec{\kappa})\rangle
$$

from which we may construct the electron density profile as

$$
\rho_{\vec{r}}=\left\langle\Phi(0)\left|a_{\vec{r}}^{\dagger} a_{\vec{r}}\right| \Phi(0)\right\rangle .
$$

Using this density, we may construct a variance tensor

$$
\sigma_{i j}^{2}=\sum_{r_{i}, r_{j}} r_{i} r_{j} \rho_{\vec{r}}
$$

in terms of which the spatial variance of the electron density in the polaron Wannier state may be given in an arbitrary direction. In the particular case of measurement along the $x$ axis in three dimensions, for example, this result after summing over the $y$ and $z$ axes takes the form

$$
\sigma_{x x}^{2}=\sum_{r_{x}, \kappa_{x} \kappa_{x}^{\prime}} \frac{r_{x}^{2}}{N_{x}^{2}} e^{-i\left(\kappa_{x}-\kappa_{x}^{\prime}\right) r_{x}}\left\langle\left\{\lambda_{\vec{q}}^{\left(\kappa_{x}, 0,0\right)}\right\} \mid\left\{\lambda_{\vec{q}}^{\left(\kappa_{x}^{\prime}, 0,0\right)}\right\}\right\rangle,
$$

where

$$
\begin{aligned}
&\left\langle\left\{\lambda_{\vec{q}}^{\left(\kappa_{x}, 0,0\right)}\right\} \mid\left\{\lambda_{\vec{q}}^{\left(\kappa_{x}^{\prime}, 0,0\right)}\right\}\right\rangle= \\
& \exp \left[-\frac{1}{N_{x} N_{y} N_{z}} \sum_{\vec{q}}\left(\frac{1}{2}\left|\lambda_{\vec{q}}^{\left(\kappa_{x}, 0,0\right)}\right|^{2}\right.\right. \\
&\left.\left.+\frac{1}{2}\left|\lambda_{\vec{q}}^{\left(\kappa_{x}^{\prime}, 0,0\right)}\right|^{2}-\lambda_{\vec{q}}^{\left(\kappa_{x}, 0,0\right) *} \lambda_{\vec{q}}^{\left(\kappa_{x}^{\prime}, 0,0\right)}\right)\right] .
\end{aligned}
$$

With further manipulation it can be shown that

$$
\sigma_{x x}^{2}=\frac{1}{N_{x}} \sum_{\kappa_{x}} \frac{1}{N_{x} N_{y} N_{z}} \sum_{\vec{q}}\left|\frac{\partial}{\partial \kappa_{x}} \lambda_{\vec{q}}^{\left(\kappa_{x}, 0,0\right)}\right|^{2} .
$$

The spatial variance of the localized electron density within the polaron is thus seen to be the average over all phonon modes and over polaron crystal momenta in the measurement direction of a mean square measure of the amount of distortion present in the the phonon amplitudes along the measurement direction.

Without further explicit calculation, this relationship provides a means of understanding what is distinct in the weak-coupling polaron behaviors found in the nonadiabatic and adiabatic regimes. In the adiabatic regime, the weak-coupling polaron band is nearly identical to that of the free electron in the inner Brillouin zone, but is strongly flattened in the outer Brillouin zone where the effects of interaction with the one-phonon continuum are severe. The phonon amplitudes exhibit strong changes in $\vec{\kappa}$ which, through 41, are associated with broad polaron Wannier states. With increasing electron-phonon coupling the severity of these $\vec{\kappa}$-dependent distortions decreases, resulting in the narrowing of the polaron Wannier state. In qualitative terms, this narrowing trend is what is expected of large polarons.

In the non-adiabatic regime however, quite a different situation is found. At weak-coupling, the polaron band is nearly identical to that of a free electron at all $\vec{\kappa}$, and in the limit of vanishing coupling is completely undistorted. The associated phonon amplitudes are not only very small, but are also very weakly distorted in $\vec{\kappa}$ which, through (41) implies the complete localization of the polaron Wannier state on a single site as $g \rightarrow 0$. Conversely, with increasing electron-phonon coupling, the presence of the higher-lying one-phonon continuum is felt more strongly at higher $\vec{\kappa}$, resulting in an enhancement of phonon amplitudes in the outer zone with which is associated a slight flattening of the polaron energy band. This growth in $\vec{\kappa}$-dependent distortion results in a broadening of the polaron Wannier state with increasing electronphonon coupling until a transition is made into the small polaron regime.

Although such compact polarons 円 are straightforwardly understood, that they are completely localized in the weak-coupling limit and broaden with increasing electron-phonon coupling suggests that they be regarded as distinct from both the large polarons and small polarons that dominate the outer reaches of the polaron parameter space. Moreover, that the compact polaron regime appears to be disconnected from both the large polaron regime and the small polaron regime by observable transition behavior suggests that such distinctions may be important to the clear classification of polaronic systems.

\section{CONCLUSION}

Our study of the Holstein model has focussed on the basic properties of observable zero-phonon lines in optical spectra; specifically, the polaron ground state energies that are principal determinants of the spectral position of such lines, and the Franck-Condon factors that are principal determinants of the oscillator strength of such lines. We have found in these results several properties that facilitate both the application of these findings to

\footnotetext{
${ }^{1}$ We use the term "compact" here to emphasize the complete spatial localization of polaron Wannier states that distinguishes the weak-coupling limit of the non-adiabatic regime from that of the adiabatic regime. This usage is at least semantically consistent with that of the theory of nonlinear waves, where particle-like excitations with finite spatial support have been termed "compactons" 35 37.
} 
experiments on real bulk materials and the interpretation of the experimental results in terms of underlying polaron structure.

First, although quantitative results can be obtained for any polaron wave vector $\vec{\kappa}$, we have found that at the extremes of isotropic polaron bands, at the Brillouin zone center and At its most remote corner, the dependence of the Franck-Condon factor on real-space dimensionality $D$ and the elementary tunneling parameter $J$ reduces to the single scaled parameter $D J$. This permits a straightforward understanding to be had of how observations in systems of reduced effective dimensionality, for example, can be expected to be related to observations in bulk systems.

Second, with some additional care, this general trend in the dependence of the Franck-Condon factors on dimensionality can be seen to be similar to that which can be expected in the $\vec{\kappa}$-dependence of the Franck-Condon factor in a system of fixed dimensionality; for example, in changing the orientation of the probed wave vector from $[1,1,1]$ to $[1,1,0]$ to $[1,0,0]$. Such experimentallycontrolled variations in the Franck-Condon factor (and its associated zero-phonon line) of a fixed system constitute a signature that can be associated with specific polaron structure.

Third, beyond such quantitative characteristics we have found that the Franck-Condon factors at the Brillouin zone center and at the extreme Brillouin zone edge constitute particularly direct means of revealing the changes in polaron structure that are associated with the self-trapping transition. That is, it appears possible to map out the polaron phase diagram from surveys utilizing Franck-Condon factors alone. Through such considerations, here extended for the first time to the non-adiabatic weak-coupling regime, we have been able to complete a systematic appraisal of polaron structure spanning all regimes. The delineation of transition curves by means of the Franck-Condon factors has compelled us to distinguish a third kind of characteristic polaron structure, the compact polaron, from the more familiar notions of the small polaron and large polaron. It is suggested that each of these three classes of polaron structure is separated from the others by an intermediate regime in which transition behavior is found in some basic polaron properties, but that this classification is essentially complete.

Thus, suitably discriminating experimental studies of the detailed behavior of zero-phonon lines would appear to afford versatile, powerful, and interpretable means of deducing the structure of polarons in real materials. Suitably constructed surveys of such structure in a variety of materials should be capable of mapping such globallyimportant features as the polaron self-trapping line, providing experimental tests of the proposed polaron phase diagram.

\section{ACKNOWLEDGEMENT}

This work was supported in part by the U.S. Department of Energy under Grant No. DE-FG03-86ER13606.

\section{APPENDIX A: DIMENSIONAL SCALING}

In some of our illustrations of specific results we take advantage of a certain scaling property that holds under the Merrifield method at the Brillouin zone center and at selected points on the Brillouin zone boundary. The demonstration of this property utilizes the fact that the Debye-Waller phases $\Phi_{\mu}^{\vec{\kappa}}$ vanish at the Brillouin zone center and everywhere on the Brillouin zone boundary, and the fact that

$$
\frac{\partial S_{\mu}^{\vec{\kappa}}}{\partial \lambda_{\vec{q}}^{\vec{\kappa}}}=-4 \frac{\lambda_{\vec{q}}^{\vec{\kappa}}}{N^{D}} S_{\mu}^{\vec{\kappa}} \sin ^{2} \frac{q_{\mu}}{2},
$$

as follows from (9).

Restricting discussion to the zone center $(\vec{\kappa}=\overrightarrow{0})$ and any of the most extreme corners of the Brillouin zone $(\vec{\kappa}=\vec{\pi})$, we find that the fundamental variational amplitudes can be expressed in the form

$$
\begin{aligned}
\lambda_{\vec{q}}^{\overrightarrow{0}} & =\frac{g \hbar \omega}{\hbar \omega-\frac{N^{D}}{\lambda_{\vec{q}}^{\overrightarrow{0}}} \frac{\partial}{\partial \lambda_{\vec{q}}^{\overrightarrow{0}}} \sum_{\mu=1}^{D} J_{\mu} S_{\mu}^{\overrightarrow{0}}}, \\
\lambda_{\vec{q}}^{\vec{\pi}} & =\frac{g \hbar \omega}{\hbar \omega+\frac{N^{D}}{\lambda_{\vec{q}}^{\vec{\pi}}} \frac{\partial}{\partial \lambda_{\vec{q}}^{\vec{\pi}}} \sum_{\mu=1}^{D} J_{\mu} S_{\mu}^{\vec{\pi}}} .
\end{aligned}
$$

Now further restricting to the isotropic case, we find

$$
\begin{aligned}
\lambda_{\vec{q}}^{\overrightarrow{0}} & =\frac{g \hbar \omega}{\hbar \omega-\frac{N^{D}}{\lambda_{\vec{q}}^{\overrightarrow{0}}} \frac{\partial}{\partial \lambda_{\vec{q}}^{\overrightarrow{0}}}\left[D J S^{\overrightarrow{0}]}\right]}, \\
\lambda_{\vec{q}}^{\vec{\pi}} & =\frac{g \hbar \omega}{\hbar \omega+\frac{N^{D}}{\lambda_{\vec{q}}^{\vec{\pi}}} \frac{\partial}{\partial \lambda_{\vec{q}}^{\vec{\pi}}}\left[D J S^{\vec{\pi}}\right]} .
\end{aligned}
$$

It is the reduction of the dimension- and $J$-dependence of the principal quantities to the simple combination $D J$ that is responsible for the simplicity of our main results.

[1] A. H. Romero, D. W. Brown, and K. Lindenberg, J. Chem. Phys. 109, 6540 (1998).

[2] T. Holstein, Ann. Phys. (N.Y.) 8, 325 (1959).

[3] T. Holstein, Ann. Phys. (N.Y.) 8, 343 (1959).

[4] M. Capone, W. Stephan, and M. Grilli, Phys. Rev. B 56, 4484 (1997-II).

[5] G. Wellein and H. Fehske, Phys. Rev. B 56, 4513 (1997).

[6] E. V. L. de Mello and J. Ranninger, Phys. Rev. B 55, 14872 (1997). 
[7] A. S. Alexandrov, V. V. Kabanov, and D. E. Ray, Phys. Rev. B 49, 9915 (1994).

[8] E. Jeckelmann and S. R. White, Phys. Rev. B 57, 6376 (1998).

[9] R. H. McKenzie, C. J. Hamer, and D. W. Murray, Phys. Rev. B 53, 9676 (1996).

[10] H. D. Raedt and A. Lagendijk, Phys. Rev. B 27, 6097 (1983).

[11] H. D. Raedt and A. Lagendijk, Phys. Rev. B 30, 1671 (1984).

[12] A. Lagendijk and H. D. Raedt, Phys. Lett. A 108A, 91 (1985).

[13] P. E. Kornilovitch, Phys. Rev. Lett. 81, 5382 (1998).

[14] A. S. Alexandrov and P. E. Kornilovitch, Phys. Rev. Lett. 82, 807 (1998).

[15] A. H. Romero, Ph.D. thesis, University of California, San Diego, 1998.

[16] D. W. Brown, K. Lindenberg, and Y. Zhao, J. Chem. Phys. 107, 3179 (1997).

[17] D. W. Brown and K. Lindenberg, Physica D 113, 267 (1998).

[18] Y. Zhao, D. W. Brown, and K. Lindenberg, J. Chem. Phys. 106, 5622 (1997).

[19] Y. Zhao, D. W. Brown, and K. Lindenberg, J. Chem. Phys. 107, 3159 (1997).

[20] A. H. Romero, D. W. Brown, and K. Lindenberg, Phys. Rev. B XX, (1999).

[21] A. H. Romero, D. W. Brown, and K. Lindenberg, Phys. Rev. B XX, to appear (1999).

[22] A. H. Romero, D. W. Brown, and K. Lindenberg, condmat 9905174 (1999).

[23] A. H. Romero, D. W. Brown, and K. Lindenberg, J. Lumin. XX, XXXX (1999).

[24] S. A. Trugman and J. Bonca, J. Supercon. 12, 221 (1999).

[25] J. Bonca, S. A. Trugman, and I. Batistic, cond-mat 9812252 (1998).

[26] A. H. Romero, D. W. Brown, and K. Lindenberg, Phys. Lett. A 254, 287 (1999).

[27] A. H. Romero, D. W. Brown, and K. Lindenberg, MRS Symp. Proc. XX, to appear (1998).

[28] A. H. Romero, D. W. Brown, and K. Lindenberg, condmat 9905148 (1999).

[29] Polarons and Excitons, edited by C. G. Cuper and G. D. Whitfield (Plenum Press, New York, 1962).

[30] V. Kenkre and P. Reineker, Exciton Dynamics in Molecular Crystals and Aggregates (Springer-Verlag, Berlin, 1982).

[31] M. Ueta, H. Kauzaki, K. Kobayashi, Y. Toyozawa, and E. Hanamura, Excitonic Processes in Solids (SpringerVerlag, Berlin, 1986).

[32] E. A. Silinsh and V. Čápek, Organic Molecular Crystals: Interaction, Localization and Transport Phenomena (AIP-Press, New York, 1994).

[33] K. S. Song and R. T. Williams, Self-Trapped Excitons (Springer Verlag, Berlin, 1996).

[34] R. E. Merrifield, J. Chem. Phys. 40, 445 (1964).

[35] P. Rosenau and J. M. Hyman, Phys. Rev. Lett. 70, 564 (1993).

[36] P. Rosenau, Phys. Rev. Lett. 73, 1737 (1994).

[37] P. Rosenau, Phys. Lett. A 211, 265 (1996). 